Case Report

\title{
Thiamine Deficiency in Self-Induced Refeeding Syndrome, an Undetected and Potentially Lethal Condition
}

\author{
Einat Hershkowitz, ${ }^{1,2}$ Alon Reshef, ${ }^{3,4}$ Olga Munich, ${ }^{2}$ Bracha Yosefi, ${ }^{2}$ and Arie Markel ${ }^{2,4}$ \\ ${ }^{1}$ Department of Clinical Nutrition, Haemek Medical Center, Afula, Israel \\ ${ }^{2}$ Department of Internal Medicine A, Haemek Medical Center, 18101 Afula, Israel \\ ${ }^{3}$ Department of Psychiatry, Haemek Medical Center, Afula, Israel \\ ${ }^{4}$ Technion, Faculty of Medicine, Haifa, Israel \\ Correspondence should be addressed to Arie Markel; arie@markel.co.il
}

Received 18 August 2014; Revised 22 November 2014; Accepted 24 November 2014; Published 28 December 2014

Academic Editor: W. Zidek

Copyright (C) 2014 Einat Hershkowitz et al. This is an open access article distributed under the Creative Commons Attribution License, which permits unrestricted use, distribution, and reproduction in any medium, provided the original work is properly cited.

\begin{abstract}
Rapid restoration of nutrients and electrolytes after prolonged starvation could result in a life threatening condition characterized by sensory and neurological dysfunction and severe metabolic imbalance that has been designated as refeeding syndrome. Its diagnosis is frequently missed resulting in severe complications and even death. We describe a 25 -years-old female patient with mental disorders and severe malnutrition who developed severe clinical manifestations and biochemical abnormalities characteristic of the refeeding syndrome, after restarting oral feeding on her own. Schizophrenia was later diagnosed. Increased awareness of this condition and its complications is necessary to prevent its detrimental complications.
\end{abstract}

\section{Introduction}

Refeeding syndrome (RFS) represents a life threatening clinical disorder that happens to occur when restarting feeding in patients with malnutrition or after prolonged starvation [1]. It was first reported among people released from concentration camps following the Second World War. Oral feeding of these severe malnourished individuals often ended in diarrhea, heart failure, neurological complications, and death [2-4].

Although this condition has been extensively described in the medical literature, it is underdiagnosed and commonly missed in everyday practice [5].

The following is the description of a young woman that was admitted to our internal medicine ward with a prolonged history of feeding problems and severe malnutrition. Attempts to improve her condition resulted in severe electrolyte imbalance and neurological problems compatible with RFS.

\section{Case Report}

A 25-year-old woman was admitted to our ward because of extreme weakness. She complained of anorexia and considerable weight loss during the last year, sometimes accompanied by nausea and vomiting of unknown cause. She had a previous mental disorder suggesting eating disturbances.

On admission she was cachectic. Her weight was $30 \mathrm{~kg}$ and the body mass index (BMI) was 12.5 (normal range: 2025).

During the first days after admission she received oral intake. She ate by herself and according to her own demands. The patient, her family, and the staff were advised regarding the recommended pace of the feeding process, based on the principle "start low, advance slow." Refeeding was done according to a feeding scheme prepared in advance. The patient was carefully observed by the nursing personnel and her family. Food was given by the nursing personnel and the patient ate small quantities by herself. During feeding she was always accompanied by her family or a nurse.

As the patient was free to eat by herself and was eager to be released, she succeeded in circumventing the recommendations around. Two days after admission at night hours, when accompanied by a member of the family, she ingested large quantity of food, relative to her regular daily intake during the previous days, in a way that suggested a "binge eating." This meal included mainly carbohydrates (simple sugars) and fats with a total caloric intake of 670 calories (49\% of 
TABLE 1: Laboratory results during hospitalization.

\begin{tabular}{|c|c|c|c|c|c|c|c|c|}
\hline Laboratory test & Normal range & $\begin{array}{l}\text { Previous } \\
\text { admission }\end{array}$ & $\begin{array}{c}\text { Day of } \\
\text { admission }\end{array}$ & $\begin{array}{l}\text { Day after the } \\
\text { eating bout }\end{array}$ & Day 2 & Day 3 & Day 8 & $\begin{array}{c}\text { Day } 29 \\
\text { Transfer to department } \\
\text { of psychiatry }\end{array}$ \\
\hline Glucose & $70-100 \mathrm{mg} / \mathrm{dL}$ & 105 & 147 & & 85 & 69 & & 74 \\
\hline Urea & $17-43 \mathrm{mg} / \mathrm{dL}$ & 8 & 118 & 26 & 10 & 13 & & 9 \\
\hline Creatinine & $0.51-0.95 \mathrm{mg} / \mathrm{dL}$ & 0.57 & 1.02 & 0.45 & 0.37 & 0.37 & & 0.32 \\
\hline Calcium & $8.5-10.5 \mathrm{mg} / \mathrm{dL}$ & 8.92 & & & 7.75 & 8.45 & & \\
\hline Phosphorus & $2.5-5 \mathrm{mg} / \mathrm{dL}$ & 3.40 & & & 0.99 & 1.16 & & 4 \\
\hline $\mathrm{TP}^{*}$ & $6.6-8.3 \mathrm{~g} / \mathrm{dL}$ & 7.6 & 7.51 & 5.61 & 5.84 & 6.44 & & 6.63 \\
\hline Albumin & $3.5-5.2 \mathrm{~g} / \mathrm{dL}$ & 4.33 & 4.69 & 3.48 & 3.56 & 3.96 & & 3.66 \\
\hline $\mathrm{Mg}^{*}$ & $1.6-2.6 \mathrm{mg} / \mathrm{dL}$ & & & & 1.58 & 1.82 & & \\
\hline $\mathrm{Na}^{*}$ & $135-145 \mathrm{mmol} / \mathrm{L}$ & 134 & 134 & 139 & 141 & 139 & & 143 \\
\hline Potassium & $3.5-5.1 \mathrm{mmol} / \mathrm{L}$ & 3.62 & 3.15 & 2.57 & 3.48 & 4.21 & & 3.76 \\
\hline Lactate & $4.5-19.8 \mathrm{mg} / \mathrm{dL}$ & & & \multicolumn{5}{|c|}{21.9} \\
\hline Vitamin B1 & $28-85 \mathrm{mg} / \mathrm{mL}$ & & & \multicolumn{5}{|c|}{16} \\
\hline Alkaline phosphatase & $30-120 \mathrm{u} / \mathrm{L}$ & 41 & 364 & 182 & 162 & 167 & & 87 \\
\hline $\mathrm{GOT}^{*}(\mathrm{AST})$ & $0-31 \mathrm{u} / \mathrm{L}$ & 36 & 1140 & 242 & 247 & 263 & & 23 \\
\hline $\mathrm{GPT}^{*}(\mathrm{ALT})$ & $0-34 \mathrm{u} / \mathrm{L}$ & 23 & 518 & 310 & 319 & 377 & & 21 \\
\hline
\end{tabular}

${ }^{*}$ TP: total protein; Mg: magnesium; Na: natrium, sodium; GOT (AST): glutamic oxaloacetic transaminase (aspartate transaminase); GPT (ALT): glutamicpyruvic transaminase (alanine transaminase).

calories from carbohydrate and $46 \%$ of calories from fat). The recommended daily caloric intake in her condition should be $20 \mathrm{kcal} / \mathrm{kg} / \mathrm{day}$, which in her case, weighing $30 \mathrm{~kg}$, should be about $600 \mathrm{kcal}$ per day. The meal she ingested that night increased the total amount of calories ingested by the patient that day to the double allowed on 24 hours.

On the day after the binge meal she complained of weakness and loss of appetite that resulted in a decrease in food intake. Her clinical condition deteriorated. She lost some cognitive abilities and showed psychiatric imbalance. She complained of headache and looked weakened and impassive, with great difficulty in understanding and in giving suitable answers to questions asked. On the same period of time there was a worsening on her legs edema. Hypokalemia was detected. Routine blood tests showed liver function tests abnormalities, electrolytes imbalance, and normal protein (total) and albumin values (Table 1). The chest XR was within normal limits and the ECG showed regular and normal rhythm.

On the second and third day after the "binge eating," severe hypophosphatemia, hypomagnesemia, and hypernatremia were noted and corrected accordingly. Clinical findings at that moment included limb edema, psychomotor agitation, headache, weakness, gastrointestinal unrest, trembling, unstable gait, and ophthalmoplegia (frozen gaze), suggesting thiamine deficiency and Wernicke's encephalopathy. The patient was started on thiamine (vitamin B1) $100 \mathrm{mg}$ orally and intravenously, based on her signs and symptoms and the laboratory results previously mentioned. Thiamine (vitamin B1) level prior to treatment was low $(16 \mathrm{mg} / \mathrm{mL})$, as later reported. Isolated episodes of hallucinations, convulsions, and syncope occurred sporadically during the next days.
Two days after starting thiamine administration her clinical condition gradually improved. There was an increase in appetite and food ingestion and a marked improvement in blood electrolytes $\left(\mathrm{K}^{+}, \mathrm{Na}^{+}\right)$and magnesium values. Blood levels of phosphorus returned to normal afterwards (Table 1).

On the course of hospitalization nutritional guidance was given to the patient and her family and they were informed about the risks involved with uncontrolled eating. In an attempt to reach control of the quantity and quality of food intake by the patient, she was advised to drink a formula of "Ensure Plus" (containing $237 \mathrm{cc} / 355 \mathrm{kcal} / 13 \mathrm{gm}$ protein/day divided into small portions during the course of the day), in addition to her usual daily intake. The hours and quantities of food ingested and the daily weight were registered by the nursing staff.

A multidisciplinary teamwork, including physicians, dietician, nursing staff, and social worker, was involved in the treatment of the patient. At the same time, her family was recruited for her support, companion, and backing and for updating and reporting on changes of behavior that could counterbalance nutritional recommendations.

Gradually she started gaining weight and after initial improvement she was transferred to the psychiatric ward. A diagnosis of schizophrenia simplex was done and olanzapine (Zyprexa) was started. Her nutritional condition, under further dietician supervision, progressively improved and on the first month after admission, she gained 4.2 kilograms of weight, representing about $12 \%$ of her body weight. On the second month of hospitalization (including her psychiatric hospitalization) she gained another $5.8 \mathrm{~kg}$ of weight. In total she regained about $10 \mathrm{~kg}$ of weight, representing about $25 \%$ of her body weight, and almost returned to her regular weight. 


\section{Discussion}

RFS is the metabolic consequence of poorly controlled refeed ing in subjects with severe malnutrition. The syndrome usually occurs after initiating guided feeding by the caregiver, especially of carbohydrates, liquids, and salt in a quantity that the cardiovascular and metabolic systems cannot hold [6].

In the present case, food was self-administered by the patient. As mentioned, this is an unexpected outcome and, to our knowledge, has not been reported before.

Manzanares and Hardy accentuated some of the main features of the RFS, including electrolyte imbalance such as hypophosphatemia, hypokalemia, and hypomagnesemia; RFS increases phosphorylation of glucose and salt and water retention leading to edema and heart failure [7].

The syndrome starts with the administration of glucose. The resultant hyperglycemia stimulates insulin secretion and triggers the intracellular process for energy production which requires phosphate. Phosphate is necessary for the generation of adenosine triphosphate from adenosine diphosphate and adenosine monophosphate and other crucial phosphorylation reactions [5]. There is activation of the $\mathrm{Na}^{+}-\mathrm{K}^{+}$ATPase transporter resulting in a shift of potassium and phosphate from the extracellular to the intracellular compartment with a consequent drop in serum electrolyte levels resulting in hypophosphatemia, hypokalemia, hypomagnesemia, and the following metabolic acidosis, ketoacidosis, and hyperosmolar states $[7,8]$.

3.1. Hypophosphatemia. Hypophosphatemia has been described in about $100 \%$ of the cases of RFS [6,9-11]. Hypophosphatemia is almost universally present in cases of RFS and has been used as a surrogate or, perhaps, as the main marker for this syndrome [6]. Intracellular and extracellular hypophosphatemia are present in RFS. As previously mentioned, phosphorus is essential for ATP formation and energy creation, especially from carbohydrate metabolism. Phosphorus deficiency could result in myriad of complications, including hemolysis, rhabdomyolysis, neurological changes, edema, cardiac decompensation, congestive heart failure $(\mathrm{CHF})$ hypotension, arrhythmias pulmonary failure, and death $[5,6,8]$.

3.2. Underdiagnosis. RFS is well described in the medical literature. Nevertheless, in the usual clinical practice, this condition is overlooked and underdiagnosed.

In the recent review previously cited [1], the authors described a large spectrum of disorders either asymptomatic or, on the contrary, presenting with severe malnutrition, that can result in RFS. The cutoff point for the diagnosis of this syndrome is arbitrary and the diagnosis is based on symptoms and signs. Therefore it is very important to look and detect serum biochemical changes for the correct diagnosis and prevention of the syndrome $[1,11]$. There are great variations in the essential features of this condition, from asymptomatic cases to those presenting with severe malnutrition, which are at risk of overt and even life threatening symptoms.
The typical presentation of this syndrome is therefore not clear cut. As pointed by Stanga et al., the full-blown syndrome should be defined by the presence of symptoms, but significant biochemical changes should be detected early in order to prevent the clinical features developing. They suggest adapting the terms "symptomatic RFS" and "potential or biochemical RFS" for the various presentations of this syndrome [1].

In 2006 a guideline was published by the National Institute for Health and Clinical Excellence (NICE) in England and Wales that used best available evidence for managing nutrition disorders, including the present condition [12].

Despite publication of these guidelines, awareness of this condition is still lacking and RFS still occurs. Following publication of the NICE recommendations, De Silva et al. surveyed medical personnel and found that only $44 \%$ of the physicians were aware of these guidelines and followed up them, in contrast to $70 \%$ of the dieticians [13]. Thirty-nine percent of the participants considered that the recommendations have to be followed up as guidance to treatment. Thirty-six percent found the recommendations too strict, whereas other participants reported that they never met a condition such as RFS [13]. The diversified point of view makes necessary further gathering of clinical data in order to set clear guidelines for treatment.

In the paper previously mentioned [9], $48 \%$ of the cases of RFS had severe malnutrition, $34 \%$ were admitted to an intensive care unit, 25\% were oncologic patients, and $9.5 \%$ had gastrointestinal fistula.

Because of the nonspecific presentation and rareness, the diagnosis of this disorder is difficult even by those familiar with these conditions. Although not all the subjects at risk will experience RFS, prediction for the specific patient that will contract this disorder is difficult [9]. There is great importance in identifying the groups at risk and the first symptoms and signs of this condition [11]. Not only are patients with extreme malnutrition, including anorexia nervosa, at high risk, but also other groups of subjects experiencing limited supply of food, psychiatric disorders, old age, or increased metabolic demands.

RFS presents usually after oral or parenteral artificial feeding $[1,11,14]$. RFS after self-administered feeding, as in the present case, is rare.

3.3. Thiamine. As pointed by Manzanares and Hardy, it is likely that thiamine may be one of the contributing factors leading to sudden death during RFS [7].

Thiamine deficiency in acute RFS can cause CHF (wet beriberi) or Wernicke's encephalopathy (WE) (dry beriberi). $\mathrm{WE}$ is an acute, neuropsychiatric syndrome characterized by acute confusional state, ataxia, hypothermia, and even coma [11].

In one of the articles previously mentioned [6], recollection of RFS cases was done during the years 20002011. The authors described 27 cases in whom the syndrome developed on the first 5 days after feeding. A routine vitamin B1 measurement was not performed. Only in one case the 
TABLE 2: Conditions associated with thiamine deficiency.

\begin{tabular}{lc}
\hline Alcoholism & Anorexia nervosa \\
Bariatric surgery & Chronic vomiting \\
Drugs: metformin, omeprazole, diuretics & Diabetes mellitus \\
Folic acid deficiency & Thiaminase \\
Chronic diarrhea & Malignancy \\
Hyperemesis gravidarum & HIV infection \\
Diet deficient in thiamine & Malabsorption \\
Ataxia type II & Refeeding syndrome \\
Megaloblastic anemia & Hyperthyroidism \\
Chemotherapeutic treatments & Systemic diseases \\
\hline
\end{tabular}

level of thiamine was determined and found to be very low, confirming the diagnosis of RFS [6].

In the case we presented, thiamine level was low, and other features typical of WE and RFS were also present.

Thiamine requirement in healthy individuals is $0.5 \mathrm{mg} /$ $1000 \mathrm{kcal}$. The vitamin reservoir could be depleted in 2-3 weeks if new supply is lacking. There are several populations with an increased risk for developing a deficit in vitamin B1. Conditions causing undernutrition are frequently related to thiamine deficiency: malabsorption secondary to surgery or gastrointestinal disease, increase in metabolic demands, and greater renal or gastrointestinal loss of nutrients, including magnesium. The most frequent cause of thiamine deficiency is alcoholism and, as frequently practiced, therapy of an alcoholic bout includes administration of vitamin B1.

Symptoms of deficiency are not specific and include frequent headache, weakness, confusion, opthalmoplegia, ataxia, abdominal discomfort, and cardiovascular manifestations as congestive heart failure $[14,15]$. These patients may go unrecognized and vitamin treatment is not implemented.

The most significant indication of thiamine deficiency is WE.

A deficit of this vitamin manifests usually after intravenous glucose administration together with increased oral carbohydrate consumption. This results in a metabolic shift from fats to carbohydrate use for energy production, a process requiring increased amounts of thiamine and leading to acute thiamine deficiency.

Conditions related to an increased risk for WE are those causing vitamin B1 deficiency, including alcohol abuse, surgical procedures of the gastrointestinal tract, recurrent vomiting or chronic diarrhea, cancer and chemotherapeutic treatments, and systemic diseases [16] (Table 2).

Therefore, it is mandatory that thiamine be given before or concomitantly with intravenous administration of glucose when risk for WE is suspected. In the present case a dose of $100 \mathrm{mg}$ of thiamine was given.

Although much higher doses are currently recommended by some organizations such as the European Federation of Neurological Societies (EFNS) and the Royal College of Physicians $[17,18]$ (200 mg intravenously 3 times daily and $500 \mathrm{mg}$ three times daily, resp.), these recommendations have not been accepted universally.
In addition, at the time the patient was given $100 \mathrm{mg}$ of thiamine, his weight and BMI were very low (30 and 12, resp.) and we were not certain of a definitive diagnosis of WE. Therefore we chose the lowest dose recommended in the literature. Furthermore, a rapid improvement and the disappearance of symptoms of WE on the following days were noted, explaining why we did not increase the dose of thiamine.

A late Cochrane report commented that the $200 \mathrm{mg}$ dose was superior to the mean result of all the other dosages, but concluded that there is insufficient evidence from randomized controlled clinical trials to guide clinicians in the dose, frequency, route, or duration of thiamine treatment for prophylaxis against, or treatment, of Wernicke-Korsakoff Syndrome (WKS) due to alcohol abuse [19]. Similarly, the EFNS guidelines, previously mentioned, reported that there is no consensus on the optimal dose of thiamine, its preparation form, duration of treatment, or the number of daily doses and that, according to many case reports, treatment with either 100 or $200 \mathrm{mg}$ thiamine given intravenously has cured the disease in the nonalcoholic. They also added that since studies of sufficient quality to warrant a formal recommendation are lacking, there is no evidence to support conclusions as to dosage, route of administration, and treatment time of thiamine [17].

As high levels of vitamin B1 are not toxic, it is recommended that the vitamin be taken uninterruptedly during several months.

In the present case we identified thiamine deficiency during RFS development as a consequence of the "binge eating" that included, as mentioned, large quantities of simple carbohydrates and fats. Worsening of symptoms in our patient occurred 24 hours after the feeding bout and progressed during the following days. Laboratory findings at that time included hypophosphatemia, hypercalcemia, hypokalemia, hypernatremia, and hypoglycemia. As the clinical presentation included features typical of WE, we decided to administer thiamine, both intravenously and orally.

Current guidelines recommend the use of magnetic resonance imaging (MRI) for the diagnosis of WE [17]. In the present case, an MRI was not performed. On the other hand, the patient in this report presented with most of the classical clinical signs in use for the diagnosis of WE. Caine et al. studied clinical features of 28 autopsy-proven alcoholic patients with WE that were well evaluated during life. They found that the sensitivity of the classic triad (eye signs, cerebellar signs, and confusion) for the diagnosis of WE was $23 \%$ but rose to $85 \%$ if the patients had at least two of the four following features: dietary deficiencies, eye signs, cerebellar signs, and either mild memory impairment or an altered mental state [20].

Our patient had all four clinical features for the diagnosis of WE previously presented. In addition, electrolyte imbalance was present and the patient responded rapidly to the treatment given. Although MRI can confirm the diagnosis, we believe that a reliable diagnosis can be done based on strong clinical features such as in the present patient.

Thiamine was administered and after a few days there was improvement in laboratory parameters and a partial recovery 
in cognition. Further improvement in physical perception and cognition occurred some days later.

\section{Conclusions}

Keep in mind the following distinctive features of RFS.

(i) High risk groups such as extreme malnutrition.

(ii) Low levels of phosphorus, magnesium, potassium, and calcium.

(iii) Low levels of vitamin B1.

(iv) Hyperglycemia/hypoglycemia.

(v) Danger for RFS being higher in the five first days of refeeding.

(vi) Keeping with the principle "start low, advance slow," when refeeding.

The reported case emphasizes the importance of identifying patients at risk to develop RFS. When acute weight loss is more than $15 \%$ of the usual weight, the risk for RFS increases and feeding has to be completed under medical supervision, even if the patient is overweight. Special attention should be given to the electrolyte disturbances, such as hypokalemia, hypophosphatemia, hypomagnesaemia, and their early identification. Thiamine levels should be measured. International guidelines suggest a daily measurement of electrolytes and administration of thiamine before refeeding.

Alertness and collaboration of a multidisciplinary professional team can prevent this disorder from happening in the population at risk. Steps for prevention of this syndrome have to be taken, such as the routine determination of serum electrolytes and phosphorus, and feeding has to be done at a slow controlled pace with special attention to details of micronutrients, such as vitamin B1, that can prevent worsening of the patient condition, forward recovery, and shorten the length of hospitalization. Vitamin B1 should be given intravenously concomitantly with IV glucose.

The question that emerges from this paper is if every patient admitted with extreme weight loss, cachexia, or drastic weight reduction should receive thiamine intravenously on the first three days of hospitalization or when starting nutrition. Should thiamine be given prior to the occurrence of the RFS, as part of a protocol in patients with increased risk for this syndrome?

This question is of crucial importance and hopefully, an answer will be given in the near future.

\section{Conflict of Interests}

The authors declare that there is no conflict of interests regarding the publication of this paper.

\section{References}

[1] Z. Stanga, A. Brunner, M. Leuenberger et al., "Nutrition in clinical practice-the refeeding syndrome: illustrative cases and guidelines for prevention and treatment," European Journal of Clinical Nutrition, vol. 62, no. 6, pp. 687-694, 2008.
[2] J. Brozek, C. B. Chapman, and A. Keys, "Drastic food restriction; effect on cardiovascular dynamics in normotensive and hypertensive conditions," The Journal of the American Medical Association, vol. 137, no. 18, pp. 1569-1574, 1948.

[3] M. A. Schnitker, P. E. Mattman, and T. L. Bliss, "A clinical study of malnutrition in Japanese prisoners of war," Annals of Internal Medicine, vol. 35, no. 1, pp. 69-96, 1951.

[4] U. G. Kyle and C. Pichard, "The Dutch Famine of 19441945: a pathophysiological model of long-term consequences of wasting disease," Current Opinion in Clinical Nutrition and Metabolic Care, vol. 9, no. 4, pp. 388-394, 2006.

[5] S. D. Hearing, "Refeeding syndrome," British Medical Journal, vol. 328, no. 7445, pp. 908-909, 2004.

[6] A. Skipper, "Refeeding syndrome or refeeding hypophosphatemia: a systematic review of cases," Nutrition in Clinical Practice, vol. 27, no. 1, pp. 34-40, 2012.

[7] W. Manzanares and G. Hardy, "Thiamine supplementation in the critically ill," Current Opinion in Clinical Nutrition \& Metabolic Care, vol. 14, no. 6, pp. 610-617, 2011.

[8] R. L. Weinsier and C. L. Krumdieck, "Death resulting from overzealous total parenteral nutrition: the refeeding syndrome revisited," The American Journal of Clinical Nutrition, vol. 34, no. 3, pp. 393-399, 1981.

[9] P. E. Marik and M. K. Bedigian, "Refeeding hypophosphatemia in critically ill patients in an intensive care unit: a prospective study," Archives of Surgery, vol. 131, no. 10, pp. 1043-1047, 1996.

[10] M. E. Hayek and P. G. Eisenberg, "Severe hypophosphatemia following the institution of enteral feedings," Archives of Surgery, vol. 124, no. 11, pp. 1325-1328, 1989.

[11] A. A. Boateng, K. Sriram, M. M. Meguid, and M. Crook, "Refeeding syndrome: treatment considerations based on collective analysis of literature case reports," Nutrition, vol. 26, no. 2, pp. 156-167, 2010.

[12] Nutrition Support for Adults: Oral Nutrition Support, Enteral Tube Feeding and Parenteral Nutrition, National Collaborating Centre for Acute Care, London, UK, 2006.

[13] A. De Silva, T. Smith, and M. Stroud, "Attitudes to NICE guidance on refeeding syndrome," British M,edical Journal, vol. 337, p. a680, 2008.

[14] J. D. C. Machado, V. M. M. Suen, F. B. Chueire, J. F. M. Marchini, and J. S. Marchini, "Refeeding syndrome, an undiagnosed and forgotten potentially fatal condition," BMJ Case Reports, 2009.

[15] K. Sriram, W. Manzanares, and K. Joseph, "Thiamine in nutrition therapy," Nutrition in Clinical Practice, vol. 27, no. 1, pp. 41$50,2012$.

[16] G. Sechi and A. Serra, "Wernicke's encephalopathy: new clinical settings and recent advances in diagnosis and management," The Lancet Neurology, vol. 6, no. 5, pp. 442-455, 2007.

[17] R. Galvin, G. Bråthen, A. Ivashynka, M. Hillbom, R. Tanasescu, and M. A. Leone, "EFNS guidelines for diagnosis, therapy and prevention of Wernicke encephalopathy," European Journal of Neurology, vol. 17, no. 12, pp. 1408-1418, 2010.

[18] A. D. Thomson, C. C. H. Cook, R. Touquet, and J. A. Henry, "The Royal College of physicians report on alcohol: guidelines for managing Wernicke's encephalopathy in the accident and emergency department," Alcohol and Alcoholism, vol. 37, no. 6, pp. 513-521, 2002. 
[19] E. Day, P. Bentham, R. Callaghan, T. Kuruvilla, and S. George, "Thiamine for Wernicke-Korsakoff Syndrome in people at risk from alcohol abuse," The Cochrane Database of Systematic Reviews, no. 1, Article ID CD004033, 2004.

[20] D. Caine, G. M. Halliday, J. J. Kril, and C. G. Harper, “Operational criteria for the classification of chronic alcoholics: identification of Wernicke's encephalopathy," Journal of Neurology Neurosurgery \& Psychiatry, vol. 62, no. 1, pp. 51-60, 1997. 


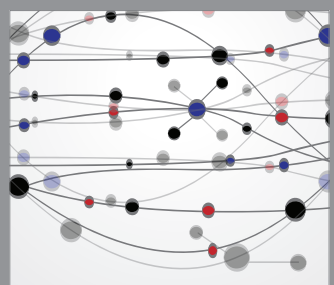

The Scientific World Journal
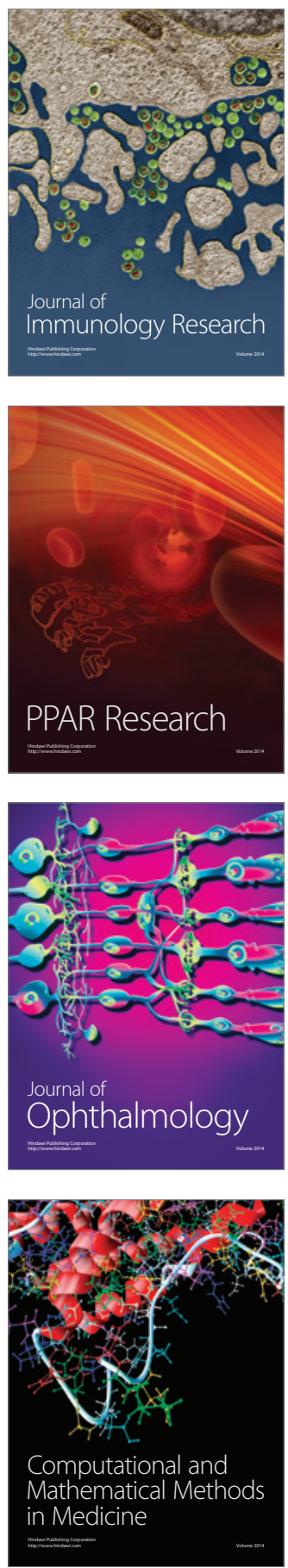

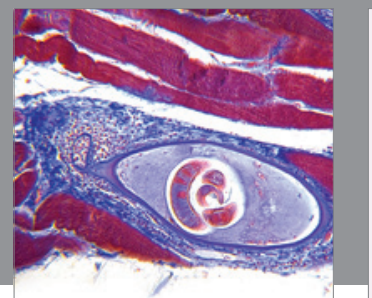

Gastroenterology

Research and Practice
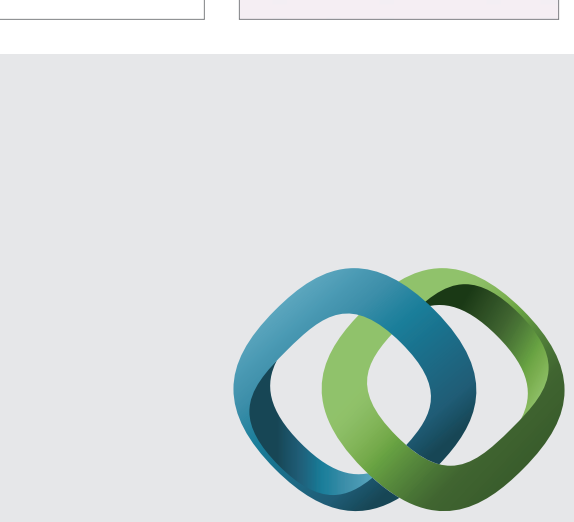

\section{Hindawi}

Submit your manuscripts at

http://www.hindawi.com
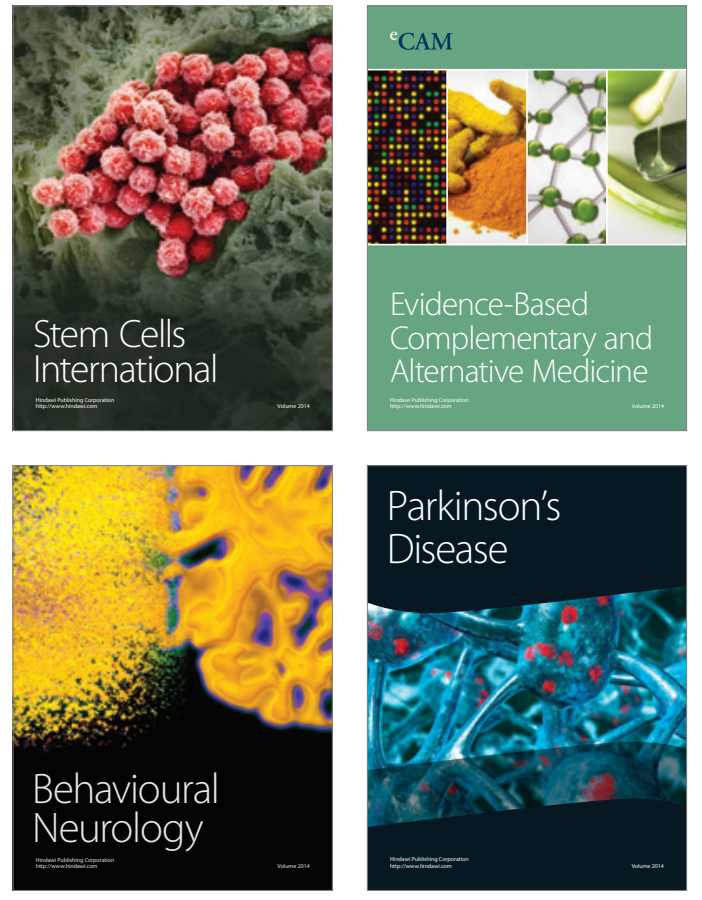
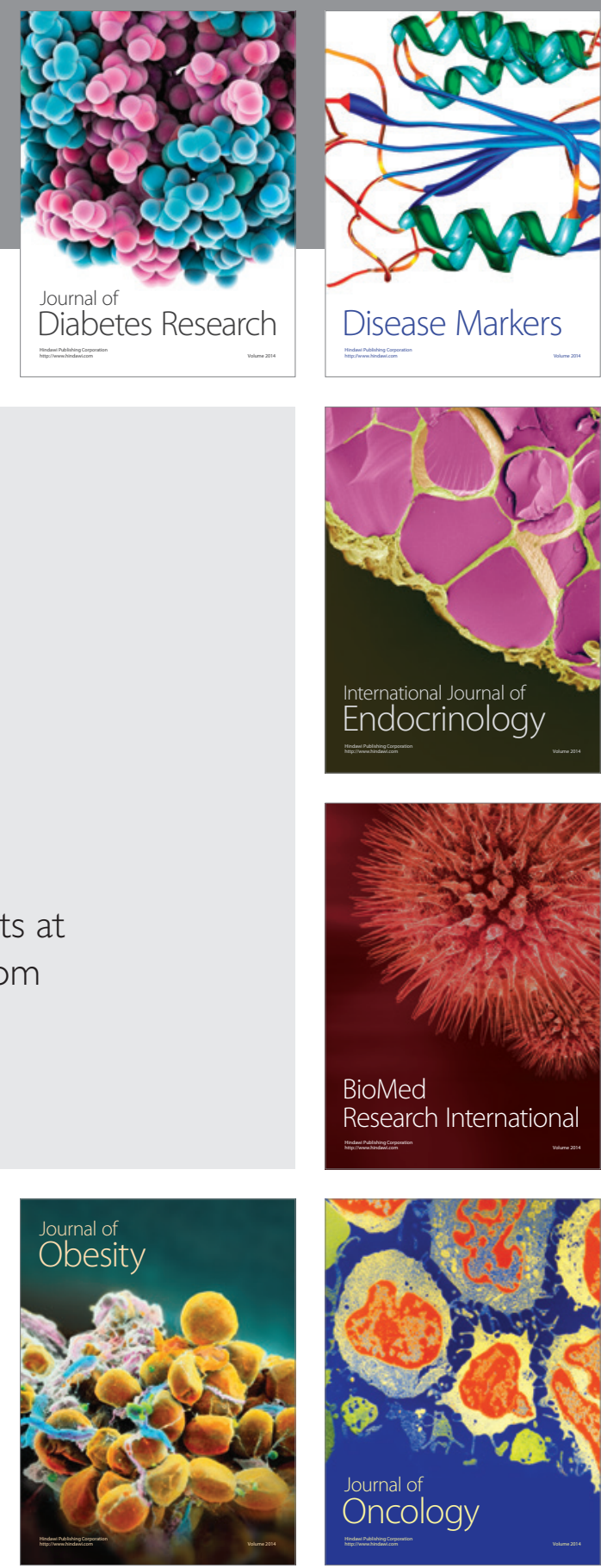

Disease Markers
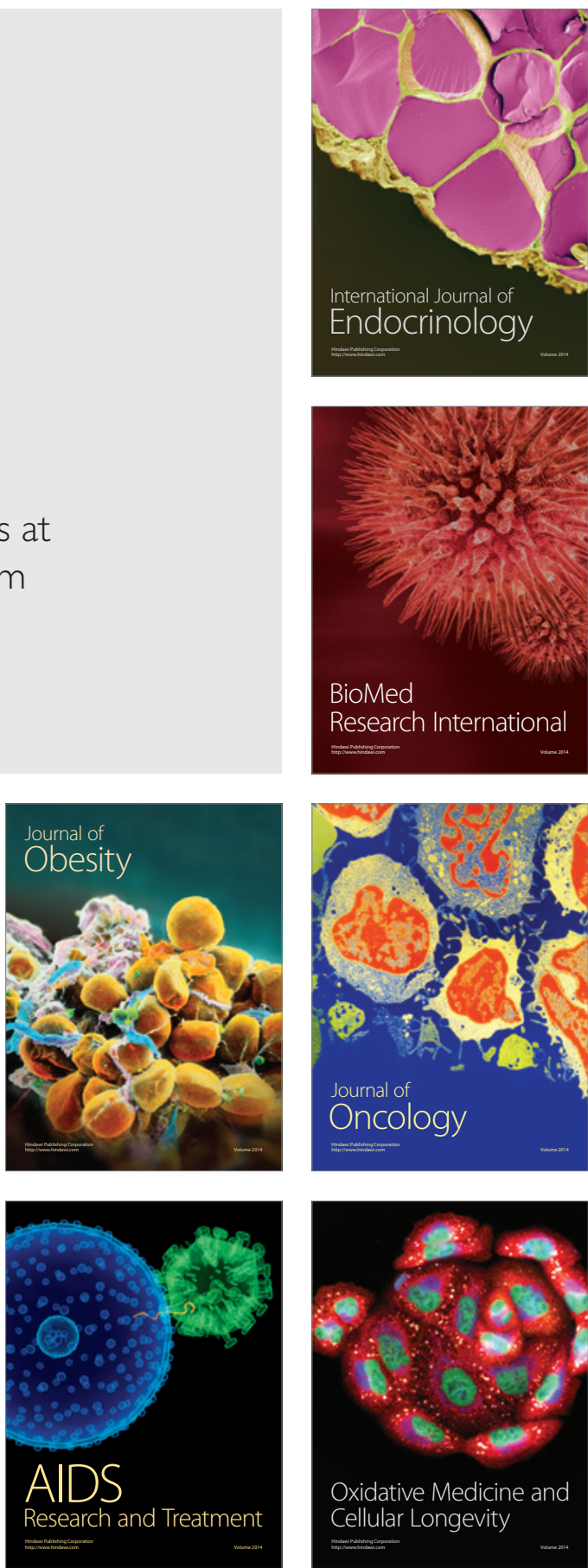